\title{
NONLINEAR PROCESSING OF HIGH RESOLUTION RADAR SEA CLUTTER
}

\author{
Mark R. Cowper and Bernard Mulgrew
}

\author{
Signals and Systems Group, Department of Electronics and Electrical Engineering, University of Edinburgh \\ The King's Buildings, Edinburgh, EH9 3JL, Scotland, UK \\ mrc@ee.ed.ac.uk, bernie@ee.ed.ac.uk
}

\begin{abstract}
Surveillance radars used at sea need to suppress the signal received back from the sea surface, in order to improve the probability of detecting target signals. This paper investigates whether nonlinear predictor networks can be used to improve the suppression of sea clutter, and thus to improve the performance of maritime surveillance radar detectors.
\end{abstract}

\section{INTRODUCTION}

The performance of surveillance radars used in marine environments is limited by sea clutter, the undesirable, but unavoidable radar returns from the sea surface. The suppression of this clutter is necessary in order to improve the target detection capability of maritime surveillance radars.

The traditional approach used to design detectors for use in sea clutter has been to use a stochastic process to model sea clutter. Evidence has suggested that the stochastic process which best models sea clutter is the compound $\mathrm{K}$ distribution, [1]. The sea clutter model is then used to choose the threshold for the radar detector, [2]. In practice, the type of radar detection technique most often applied is some form of the constant false alarm rate (CFAR) detector [3-6].

Recently, an argument has been put forward, which suggests that sea clutter is not in fact a stochastic process, but rather, it is a chaotic process [7-10]. If sea clutter is chaotic, then a nonlinear detector will exploit this property. If it is not, a nonlinear detector may exploit the non-Gaussian nature of high resolution sea clutter. The purpose of this paper is to investigate whether nonlinear predictor networks can be used to improve high resolution radar target detection at sea, using the sea clutter data sets listed in Table 1.

\section{SEA CLUTTER DATA}

Sea clutter data sets have been collected using stationary, land-based radars that operate in a dwelling mode, that is, with the antenna pointing towards a patch of the sea surface

This work was supported by Marconi Avionics, DERA, and EPSRC.

\begin{tabular}{||c|c|l||}
\hline Name & Frequency & Description \\
\hline Wavetank & $15.75 \mathrm{GHz}$ & $\begin{array}{l}\text { Data collected for a range of } \\
\text { controlled windspeeds corresponding } \\
\text { to sea states from 2 to 6. Horizontal } \\
\text { polarisation, pulse repetition } \\
\text { frequency of } 1 \mathrm{kHz} .\end{array}$ \\
\hline Dawber-H & $3 \mathrm{GHz}$ & $\begin{array}{l}\text { Data collected during a sea } \\
\text { state of } 7 \text {. Horizontal polarisation, } \\
\text { pulse repetition frequency of 20kHz. }\end{array}$ \\
\hline Dawber-V & $3 \mathrm{GHz}$ & $\begin{array}{l}\text { Data collected during a sea state of } 6 . \\
\text { Vertical polarisation, pulse } \\
\text { repetition frequency of 20kHz. }\end{array}$ \\
\hline
\end{tabular}

Table 1: Sea clutter data

along a fixed direction. Table 1 summarises the clutter data analysed for this paper.

\section{PREDICTION}

The radial basis function (RBF) network and the Volterra series network have been chosen to implement the nonlinear predictors. These networks are briefly discussed below.

\subsection{Prediction problem}

In its simplest form, a prediction problem is based on a time series $\{x(n)\}$. Given an embedding vector $\mathbf{x}(n)$, which contains $\mathrm{N}$ consecutive samples of the time series, i.e. :

$$
\mathbf{x}(n)=[x(n), x(n-1), \ldots, x(n-N+1)]^{T}
$$

This is used to form an estimate $\hat{x}(n+1)$ of the next data sample, $x(n+1)$, by constructing a predictor function $f()$, where

$$
\hat{x}(n+1)=f(\mathbf{x}(n))
$$




\subsection{RBF predictor}

An RBF network can be used to find the predictor function discussed above. The structure of the RBF predictor is shown in Figure 1. It consists of $N$ source nodes, $M$ centres (or hidden units), and $M$ weights.

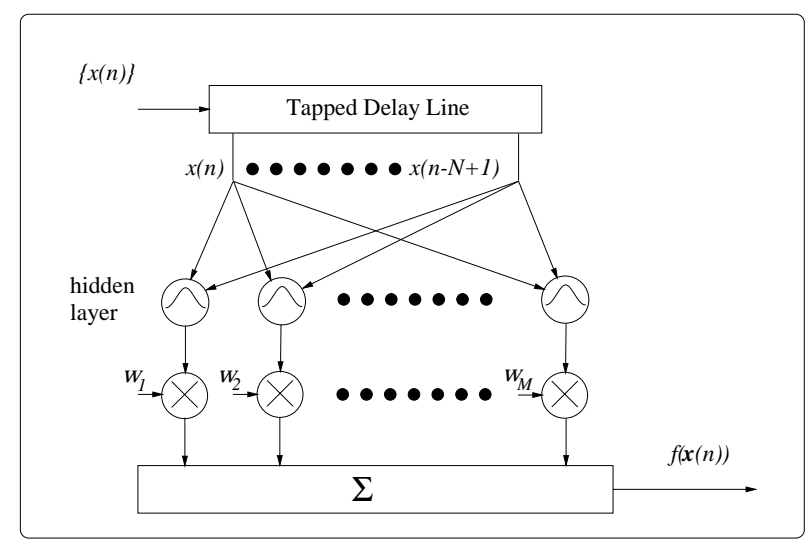

Figure 1: RBF network

The RBF predictor function is given by, (1), below:

$$
f(\mathbf{x}(n))=\sum_{i=1}^{M} w_{i} \phi\left(\left\|\mathbf{x}(n)-\mathbf{c}_{i}\right\|\right)
$$

where $M$ is the number of centres, $\phi()$ is some non-linear function, $\|$.$\| is a distance measure, \mathbf{c}_{i}$ is the position of the $i^{t h}$ centre in $N$-dimensional space, and $w_{i}$ is the weight at the output of the $i^{\text {th }}$ centre.

The nonlinear function chosen for this work was the Gaussian function, which is symbolised in Figure 1 by the bell-shaped curves in the hidden layer. Normalised, [11], as well as un-normalised Gaussian kernels have been used. The distance measure used was the Euclidean distance.

\subsection{Volterra series predictor}

The truncated Volterra series, (2), can also be used to find the predictor function discussed above.

$$
\begin{aligned}
y(n) & =\sum_{m_{1}=0}^{N-1} h_{1}\left(m_{1}\right) x\left(n-m_{1}\right) \\
& +\sum_{m_{1}=0}^{N-1} \sum_{m_{2}=m_{1}}^{N-1} h_{2}\left(m_{1}, m_{2}\right) x\left(n-m_{1}\right) x\left(n-m_{2}\right)+\ldots \\
& +\sum_{m_{1}=0}^{N-1} \ldots \sum_{m_{P}=m_{1}}^{N-1} h_{2}\left(m_{1}, m_{2}, \ldots, m_{P}\right) \\
& \times x\left(n-m_{1}\right) \ldots x\left(n-m_{P}\right)
\end{aligned}
$$

The truncated Volterra series expansion is given above, for a zero mean process. $N$ is the embedding dimension, $P$ is the order of the Volterra series, and $h($.$) is known as a Volterra$ kernel.

\subsection{Nonlinear network parameters}

The weights for the linear and RBF predictors, and the Volterra kernels were all trained using the numerically robust Householder transform, [12]. The RBF centres were selected using a random subset of the training data.

\section{DETECTION STRATEGIES}

A standard fixed threshold detector and a linear predictordetector have been used to compare the performance of the nonlinear predictor-detectors.

\subsection{False alarm rate}

A false alarm refers to noise or clutter crossing the detector threshold level, and being mistaken for a target signal. In practice, radar detectors are designed by choosing an acceptable false alarm rate (FAR), and then setting the threshold level of the detector for this FAR.

\subsection{Fixed threshold detector}

A threshold was set for this detector using a small set of clutter-only data, to form a clutter amplitude histogram. The histogram was used to determine a threshold level that would result in a tolerable number of false alarms. This threshold level was then fixed and radar data, other than that used to form the histogram, was used to judge the performance of the detector.

\subsection{Predictor-detectors}

A block diagram of the general structure of the predictordetectors is given below. The predictor (linear, RBF, or Volterra series) section was trained using a small set of clutteronly data. After training, the free parameters of the predictor were fixed. Another small set of clutter-only data was then passed through the predictor, and the errors produced were used to set an error threshold for a desired FAR. Radar data previously unseen by the detector was then used to assess the predictor-detector's performance. The idea is that during training the predictor should learn to recognise the clutter. Therefore, if clutter-only data is present at the input, the predictor will produce a small error. If a target as well as clutter is present at the input, then the predictor will produce a large error. 


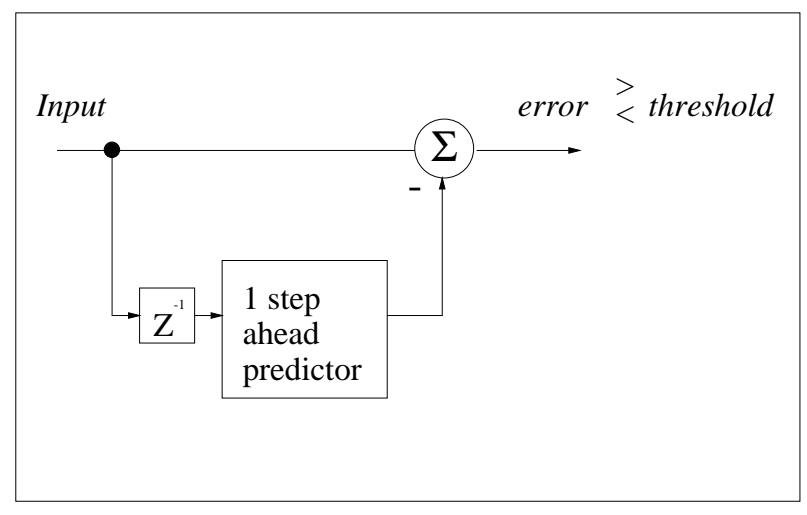

Figure 2: Predictor-detector structure

\section{RESULTS}

Prediction and detection results are presented for the sea clutter data listed in Table 1. To obtain the predictor and predictor-detector results, in sections 5.1 and 5.2, one stepahead predictors were used on clutter amplitude data.

\subsection{Prediction results}

Prediction results were obtained using 6000 samples to train the predictor's weights, then a further 6000 samples were used to obtain the normalised mean square error (NMSE) results:

$$
\mathrm{NMSE}=10 \log _{10}\left(\frac{1}{\sigma_{y}^{2} N} \sum_{k=1}^{N}(y(k)-\hat{y}(k))^{2}\right)
$$

where $y(k)$ is the actual sample, $\hat{y}(k)$ is the predictor's estimate of $y(k)$, and $\sigma_{y}^{2}$ is the variance of $y$ over the test duration, $N$.

\subsubsection{Prediction results for Wavetank data}

As listed in Table 1, Wavetank data was recorded for a range of controlled windspeeds. This data was collected using horizontal polarisation both on transmit and receive. Some results for this data are reported below.

Figure 3 shows results for a linear predictor with a range of tap lengths. Figure 3 also shows results for an RBF predictor with normalised Gaussian kernels, and an embedding dimension of 10. The number of centres used in the RBF predictor was varied from 10 to 400 . Figure 4 shows results for a cubic Volterra series predictor, for a range of embedding dimensions.

Both Figure 3 and Figure 4 show that the clutter data set recorded during a windspeed of $4 \mathrm{~m} / \mathrm{s}$ is barely predictable. The $11 \mathrm{~m} / \mathrm{s}$ and $12 \mathrm{~m} / \mathrm{s}$ data sets are more predictable than the $4 \mathrm{~m} / \mathrm{s}$ data set. In common with this increase in predictability, the authors noticed an increase in the "spikyness" of the clutter received by the radar, as the windspeed was

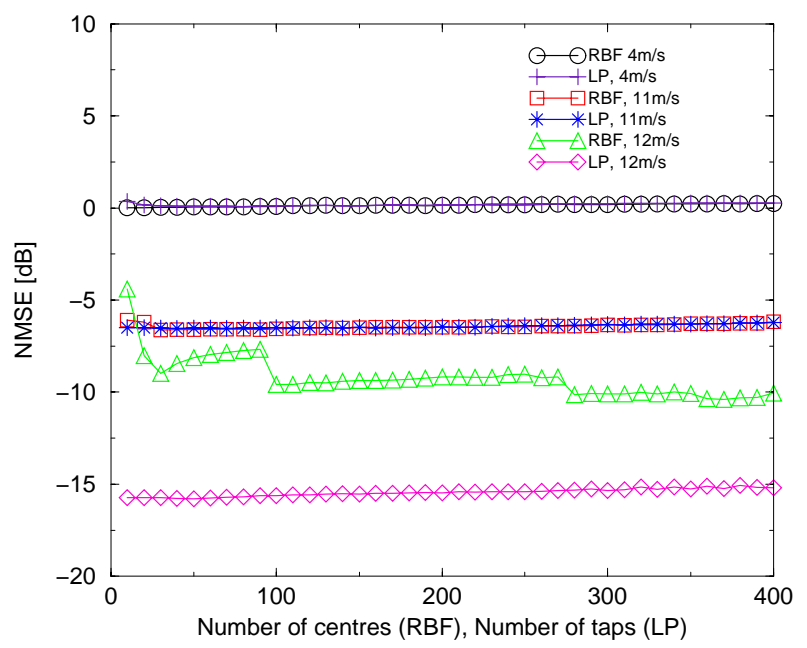

Figure 3: RBF and Linear prediction of Wavetank data

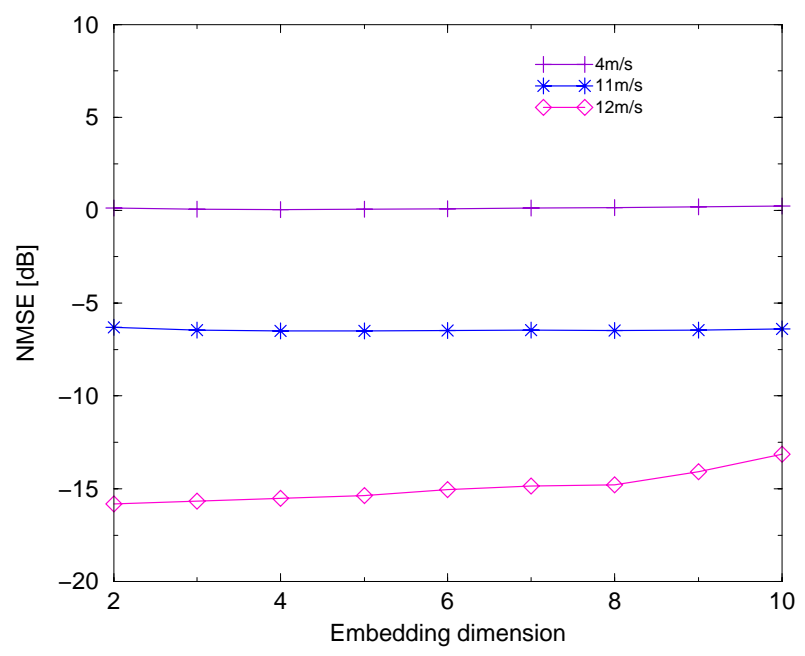

Figure 4: Volterra series prediction of Wavetank data

increased. Figures 3 and 4 show that the performance of the linear predictor is at least as good as the performance of the nonlinear predictors. These results suggest that the predictor function of the Wavetank data is linear.

\subsubsection{Prediction results for sea data}

Results presented here are for those data sets listed in Table 1, other than the Wavetank data. Figure 5 shows linear and RBF prediction results. The RBF embedding dimension was 5, and normalised Gaussian kernels were used. Figure 6 shows results for a cubic Volterra series predictor. As seen for the Wavetank data in Figure 3 and Figure 4, the linear predictor works at least as well as the nonlinear predictors, suggesting that the predictor function for these clutter data sets is also linear. Interestingly, the Dawber 


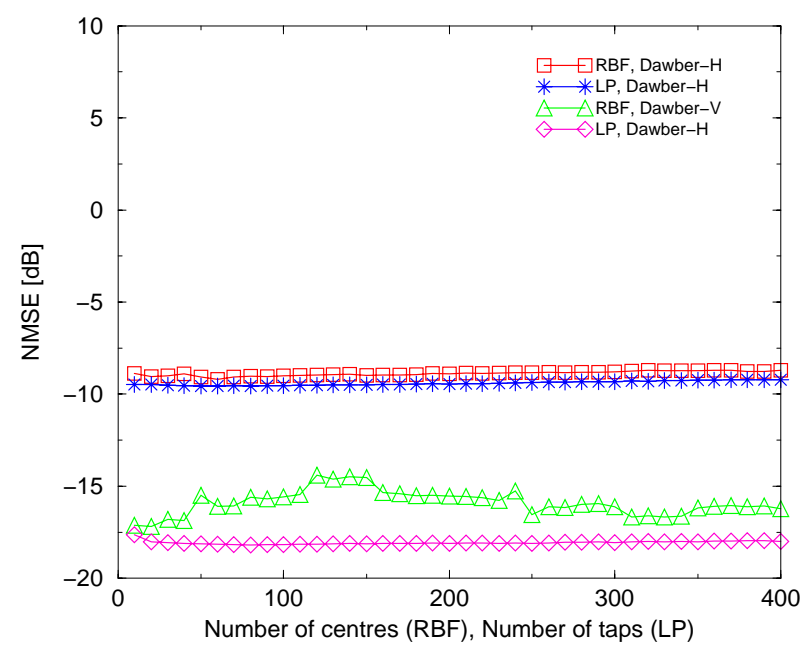

Figure 5: RBF and Linear prediction of sea data

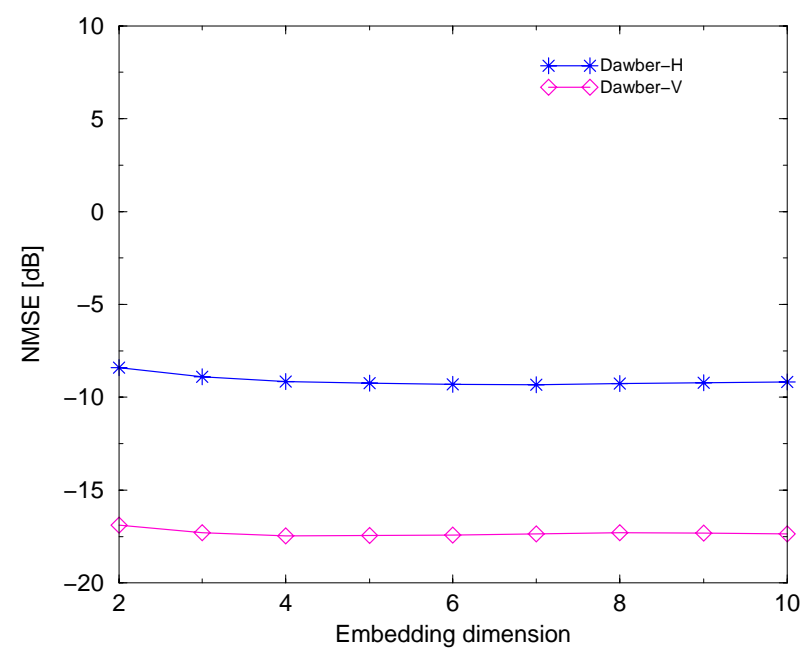

Figure 6: Volterra series prediction of sea data

data which was collected using vertical (Dawber-V) polarisation (on transmit and receive) is more predictable than that collected using horizontal (Dawber-H) polarisation (on transmit and receive), despite the fact that the data collected using horizontal polarisation was collected during a higher (windspeed) sea state. The horizontally polarised Dawber data was recorded during a windspeed of $15.4 \mathrm{~m} / \mathrm{s}$, whilst the vertically polarised Dawber data was collected during a windspeed of $12.8 \mathrm{~m} / \mathrm{s}$. All reported parameters, apart from windspeed and polarisation, were approximately the same for both Dawber data sets.

\subsection{Detection results}

Figure 7 and Figure 8 below show receiver operating curves (ROC's) for the $12 \mathrm{~m} / \mathrm{s}$ and $4 \mathrm{~m} / \mathrm{s}$ Wavetank data sets, re- spectively. In each case a Swerling, [2], (fluctuating Rayleigh) target was used, and the signal to clutter ratio was set to $0.2 \mathrm{~dB}$. In both plots an embedding dimension of 10 was used for both the Volterra, and the linear predictordetector. A set of 6000 samples was used to set the threshold level for the fixed threshold detector.

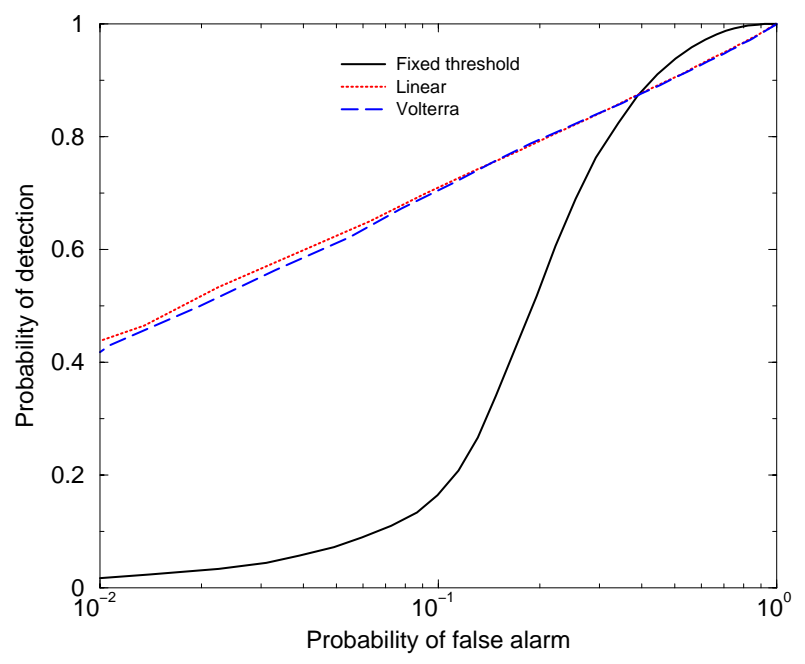

Figure 7: Detection of 12m/s Wavetank data

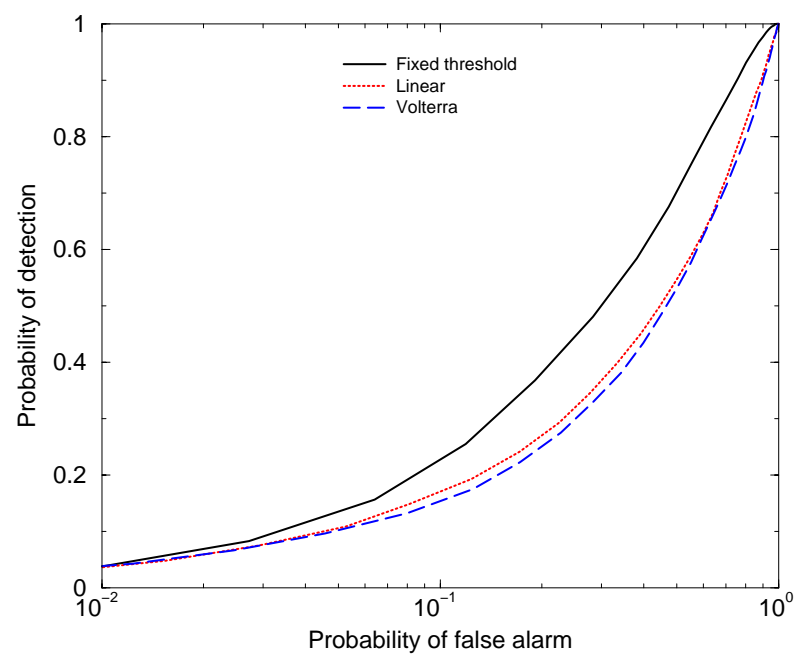

Figure 8: Detection of $4 \mathrm{~m} / \mathrm{s}$ Wavetank data

The results in Figure 7 and Figure 8 reinforce the prediction results in Figure 3 and Figure 4, which suggest that the predictor function for the Wavetank data is linear. It is observed that the detection performance of the linear predictor-detector is very similar to that of the nonlinear predictor-detector. The predictor-detectors perform more poorly than the fixed threshold level detector on the barely predictable $4 \mathrm{~m} / \mathrm{s}$ Wavetank data. However, on the more pre- 
dictable $12 \mathrm{~m} / \mathrm{s}$ data, the predictor-detectors performed better than the fixed threshold detector, for low probabilities of false alarm.

\section{CONCLUSIONS}

The predictor-detectors have been shown to perform better than a fixed threshold detector in predictable clutter. However, the results which have been presented suggest that the clutter data sets analysed for this paper have linear, rather than nonlinear, predictor functions. Therefore, the nonlinear predictor-detectors provide no additional performance benefits with respect to those obtained using a linear predictor-detector.

The effect of windspeed on sea clutter predictability has also been investigated. Data sets recorded during higher windspeed conditions appear to result in more predictable clutter, given that all other parameters remain constant, such as radar polarisation.

\section{ACKNOWLEDGEMENT}

The authors of this paper would like to express their gratitude to the Defence Evaluation and Research Agency, UK, for providing the sea clutter data listed in Table 1.

\section{REFERENCES}

[1] K. D. Ward, "Compound representation of high resolution sea clutter," IEE Electronics Letters, vol. 17, no. 16, pp. 561$563,1981$.

[2] M. I. Skolnik, Introduction to radar systems. McGraw-Hill, 1981.

[3] S. Watts, "Radar detection prediction in sea clutter using the compound k-distribution model," IEE Proceedings-F, vol. 132, pp. 613-619, December 1985.

[4] S. Watts, C. J. Baker, and K. D. Ward, "Maritime surveillance radar part 2: Detection performance prediction in sea clutter," IEE Proceedings-F, vol. 137, pp. 63-72, April 1990.

[5] S. Watts, "Cell-averaging CFAR gain in spatially correlated K-distributed clutter,' IEE Proceedings-F, vol. 143, pp. 321327, October 1996.

[6] W. M. Denny, "K-distributed sea clutter: Performance predictions made easy," in IEE Radar '97, (Edinburgh, Scotland), pp. 209-213, October 1997.

[7] H. Leung and S. Haykin, "Is there a radar clutter attractor?," Applied Physics Letters, vol. 56, no. 6, 1990.

[8] S. Haykin and S. Puthusserypady, "Chaotic dynamics of sea clutter: an experimental study," in IEE Radar '97, (Edinburgh, Scotland), pp. 75-79, October 1997.

[9] S. Haykin and S. Puthusserypady, "Chaotic dynamics of sea clutter," Choas, vol. 7, no. 4, pp. 777-802, 1997.
[10] S. Haykin and B. X. Li, "Detection of signals in chaos," Proceedings of the IEEE, vol. 83, pp. 95-122, January 1995.

[11] M. Benaim, "On functional approximation with normalized gaussian units," Neural Computation, vol. 6, pp. 319-333, 1994.

[12] A. O. Steinhardt, "Householder transforms in signal processing," IEEE ASSP Magazine, pp. 4-12, 1988. 